\title{
Orthogonal Matching Pursuit for Sparse Signal Recovery
}

\author{
T. Tony Cai ${ }^{1}$ and Lie Wang ${ }^{2}$ \\ University of Pennsylvania and Massachusetts Institute of Technology
}

\begin{abstract}
We consider the orthogonal matching pursuit (OMP) algorithm for the recovery of a high-dimensional sparse signal based on a small number of noisy linear measurements. OMP is an iterative greedy algorithm that selects at each step the column which is most correlated with the current residuals. In this paper, we present a fully data driven OMP algorithm with explicit stopping rules. It is shown that under conditions on the mutual incoherence and the minimum magnitude of the nonzero components of the signal, the support of the signal can be recovered exactly by the OMP algorithm with high probability. In addition, we also consider the problem of identifying significant components in the case where some of the nonzero components are possibly small. It is shown that in this case the OMP algorithm will still select all the significant components before possibly selecting incorrect ones. Moreover, with modified stopping rules, the OMP algorithm can ensure that no zero components are selected.
\end{abstract}

Keywords: Compressed sensing, $\ell_{1}$ minimization, mutual incoherence, orthogonal matching pursuit, signal reconstruction, support recovery.

\footnotetext{
${ }^{1}$ Department of Statistics, The Wharton School, University of Pennsylvania, Philadelphia, PA 19104, USA; e-mail: tcai@wharton.upenn.edu. Research supported in part by NSF FRG Grant DMS-0854973.

${ }^{2}$ Department of Mathematics, Massachusetts Institute of Technology, Cambridge, MA 02139, USA; e-mail: liewang@math.mit.edu. Research supported by NSF Grant DMS-1005539.
} 


\section{Introduction}

Recovery of a high-dimensional sparse signal based on a small number of linear measurements, possibly corrupted by noise, is a fundamental problem in signal processing. Specifically one considers the following model:

$$
y=X \beta+\epsilon,
$$

where the observation $y \in \mathbb{R}^{n}$, the matrix $X \in \mathbb{R}^{n \times p}$, and the measurement errors $\epsilon \in$ $\mathbb{R}^{n}$. Suppose $X=\left(X_{1}, X_{2}, \cdots, X_{p}\right)$ where $X_{i}$ denotes the $i$ th column of $X$. Throughout the paper we shall assume that the columns of $X$ are normalized, i.e. $\left\|X_{i}\right\|_{2}=1$ for $i=1,2, \cdots, p$. The goal is to reconstruct the unknown vector $\beta \in \mathbb{R}^{p}$ based on $y$ and $X$. A setting that is of significant interest and challenge is when the dimension $p$ of the signal is much larger than the number of measurements $n$. This and other related problems have received much recent attention in a number of fields including applied mathematics, electrical engineering, and statistics.

For a vector $\beta=\left(\beta_{1}, \cdots, \beta_{p}\right) \in \mathbb{R}^{p}$, the support of $\beta$ is defined to be the $\operatorname{set} \operatorname{supp}(\beta)=$ $\left\{i: \beta_{i} \neq 0\right\}$ and $\beta$ is said to be $k$-sparse if $|\operatorname{supp}(\beta)| \leq k$. A widely used framework for sparse signal recovery is the Mutual Incoherence Property (MIP) introduced in Donoho and Huo (2001). The mutual incoherence is defined by

$$
\mu=\max _{i \neq j}\left|\left\langle X_{i}, X_{j}\right\rangle\right|
$$

The MIP requires the mutual incoherence $\mu$ to be small. Other conditions used in the compressed sensing literature include the Restricted Isometry Property (RIP) and Exact Recovery Condition (ERC). See, for example, Candes and Tao (2005) and Tropp (2004). In contrast to the MIP, these conditions are not computationally feasible to verify for a given matrix $X$. 
In the present paper we consider the orthogonal matching pursuit (OMP) algorithm for the recovery of the support of the $k$-sparse signal $\beta$ under the model (1). OMP is an iterative greedy algorithm that selects at each step the column of $X$ which is most correlated with the current residuals. This column is then added into the set of selected columns. The algorithm updates the residuals by projecting the observation $y$ onto the linear subspace spanned by the columns that have already been selected, and the algorithm then iterates. Compared with other alternative methods, a major advantage of the OMP is its simplicity and fast implementation. This method has been used for signal recovery and approximation, for example, in Davis, Mallat, and Avellaneda (1997), Tropp (2004, 2006), and Barron et al. (2008). In particular, support recovery has been considered in the noiseless case by Tropp (2004), where it was shown that $\mu<\frac{1}{2 k-1}$ is a sufficient condition for recovering a $k$-sparse $\beta$ exactly in the noiseless case. Results in Cai, Wang and $\mathrm{Xu}$ (2010a) imply that this condition is in fact sharp.

In this paper we consider the OMP algorithm in the general setting where noise is present. Note that the residuals after each step in the OMP algorithm are orthogonal to all the selected columns of $X$, so no column is selected twice and the set of selected columns grows at each step. One of the key components of an iterative procedure like OMP is the stopping rule. Specific stopping rules are given for the OMP algorithm in both bounded noise and Gaussian noise cases. The algorithm is then fully data-driven. Our results show that under the MIP condition $\mu<\frac{1}{2 k-1}$ and a condition on the minimum magnitude of the nonzero coordinates of $\beta$, the support of $\beta$ can be recovered exactly by the OMP algorithm in the bounded noise cases and with high probability in the Gaussian case. In fact, it can be seen from our discussion in Section 3 that a more general condition than $\mu<\frac{1}{2 k-1}$ can guarantee the recovery of the support with high probability. In particular, all the main results hold under the Exact Recovery Condition (ERC). 
In many applications, the focus is often on identifying significant components, i.e., coordinates of $\beta$ with large magnitude, instead of the often too ambitious goal of recovering the whole support of $\beta$ exactly. In this paper, we also consider the problem of identifying large coordinates of $\beta$ in the case where some of the nonzero coordinates are possibly small. It is shown that in this case the OMP algorithm will still select all the most important components before possibly selecting incorrect ones. In addition, with modified stopping rules, the OMP algorithm can ensure that no zero components are selected.

Besides OMP, several other methods for sparse signal recovery have been proposed and extensively studied in the literature. In particular, it is now well understood that $\ell_{1}$ minimization methods provide effective ways for reconstructing a sparse signal. For example, the $\ell_{1}$ penalized least squares (Lasso) estimator has been studied in Tibshirani (1996), Chen, Donoho and Saunders (1998), and Efron et al. (2004). Zhao and Yu (2006) considered support recovery using the Lasso. In addition, two specific constrained $\ell_{1}$ minimization methods have been well studied. Donoho, Elad, and Temlyakov (2006) considered constrained $\ell_{1}$ minimization under an $\ell_{2}$ constraint. Candes and Tao (2007) introduced the Dantzig Selector, which is a constrained $\ell_{1}$ minimization method under an $\ell_{\infty}$ constraint. A particularly simple and elementary analysis of constrained $\ell_{1}$ minimization methods is given in Cai, Wang, and Xu (2010b). Bickel, Ritov and Tsybakov (2009) gives a unified treatment of the Lasso and Dantzig Selector.

Compare with the known results on the model selection consistency of the Lasso in the Gausian noise case given in Zhao and Yu (2006), the condition on the minimum magnitude of the nonzero coordinates of $\beta$ is much weaker for OMP than for the Lasso. More detailed discussion can be found in Section 3. This together with the computational simplicity make OMP a very appealing method for support recovery.

The rest of the paper is organized as follows. We will begin in Section 2 with a detailed 
description of the OMP algorithm. The stopping rules and the properties of the algorithm are considered in Section 3 for both bounded noise cases and Gaussian noise case. The theoretical results are first formulated under the MIP. Section 4 discusses the corresponding results under the ERC and compares our results with some of existing ones in the literature. Section 5 provides some technical analysis of the OMP algorithm which sheds light on how and when the OMP algorithm works properly. The proofs of the main results are contained in Section 6.

\section{The OMP Algorithm}

In this section we give a detailed description of the orthogonal matching pursuit (OMP) algorithm. We assume that the columns of $X$ are normalized so that $\left\|X_{i}\right\|_{2}=1$ for $i=$ $1,2, \cdots, p$. For any subset $S \subseteq\{1,2, \ldots, p\}$, denote by $X(S)$ a submatrix of $X$ consisting of the columns $X_{i}$ with $i \in S$. In this paper we shall also call columns of $X$ variables by following the convention in statistics. Thus we use $X_{i}$ to denote the both $i$ th column of $X$ and the $i$ th variable of the model. Following the same convention, we shall call $X_{i}$ a correct variable if the corresponding $\beta_{i} \neq 0$ and call $X_{i}$ an incorrect variable otherwise. With slight abuse of notation, we shall use $X(S)$ to denote both the subset of columns of $X$ with indices in $S$ and the corresponding submatrix of $X$.

The OMP algorithm can be stated as follows.

- Step 1: Initialize the residual $r_{0}=y$ and initialize the set of selected variable $X\left(c_{0}\right)=$ $\emptyset$. Let iteration counter $i=1$.

- Step 2: Find the variable $X_{t_{i}}$ that solves the maximization problem

$$
\max _{t}\left|X_{t}^{\prime} r_{i-1}\right|
$$

and add the variable $X_{t_{i}}$ to the set of selected variables. Update $c_{i}=c_{i-1} \cup\left\{t_{i}\right\}$. 
- Step 3: Let $P_{i}=X\left(c_{i}\right)\left(X\left(c_{i}\right)^{\prime} X\left(c_{i}\right)\right)^{-1} X\left(c_{i}\right)^{\prime}$ denote the projection onto the linear space spanned by the elements of $X\left(c_{i}\right)$. Update $r_{i}=\left(I-P_{i}\right) y$.

- Step 4: If the stopping condition is achieved, stop the algorithm. Otherwise, set $i=i+1$ and return to Step 2 .

The OMP is a stepwise forward selection algorithm and is easy to implement. A key component of OMP is the stopping rule which depends on the noise structure. In the noiseless case the natural stopping rule is $r_{i}=0$. That is, the algorithm stops whenever $r_{i}=0$ is achieved. In this paper, we shall consider several different noise structures. To be more specific, two types of bounded noise are considered. One is $\ell_{2}$ bounded noise, i.e., $\|\epsilon\|_{2} \leq b_{2}$ for some constant $b_{2}>0$. Another is $\ell_{\infty}$ bounded noise where $\left\|X^{\prime} \epsilon\right\|_{\infty} \leq b_{\infty}$ for some constant $b_{\infty}>0$. In addition, we shall also consider the important case of Gaussian noise where $\epsilon_{i} \stackrel{i i d}{\sim} N\left(0, \sigma^{2}\right)$. The stopping rule for each case and the properties of the resulting procedure will be discussed in Section 3.

\section{The OMP Algorithm: Stopping Rules and Properties}

In this section we discuss the stopping rules and investigate the properties of the OMP algorithm for the bounded noise cases as well as the Gaussian noise case. Results for the noiseless case can be found in Tropp (2004).

We begin with the basic notation and definitions. The mutual incoherence of $X$, defined in (2), is the maximum magnitude of the pairwise correlation between the columns of $X$. Let $T=\left\{i: \beta_{i} \neq 0\right\}$ be the support of $\beta$ and let $X(T)$ be the set of columns of $X$ corresponding to the support $T$. Define

$$
M=\max _{x \in X \backslash X(T)}\left\{\left\|\left(X(T)^{\prime} X(T)\right)^{-1} X(T)^{\prime} x\right\|_{1}\right\}
$$

The condition 
is called the Exact Recovery Condition (ERC) in Tropp (2004). It was shown in Tropp (2004) that the ERC is a sufficient condition for the exact recovery of the support of the signal $\beta$ in the noiseless case. It is easy to see that the value of $M$ is not computable as it depends on the unknown support $T$. However, it can be easily bounded in terms of the mutual incoherence $\mu$.

Lemma 1 If $\mu<\frac{1}{2 k-1}$, then $M \leq \frac{k \mu}{1-(k-1) \mu}<1$.

This lemma is a special case of Theorem 3.5 in Tropp (2004). The extreme eigenvalues of $X(T)^{\prime} X(T)$ are also useful. Denote the minimum and maximum eigenvalues of $X(T)^{\prime} X(T)$ by $\lambda_{\min }$ and $\lambda_{\max }$ respectively. The minimum eigenvalue $\lambda_{\min }$ is a key quantity to the sparse signal recovery problem. It has been used in, for example, Zhao and Yu (2006) and Cai, Wang and $\mathrm{Xu}(2010 \mathrm{~b})$. Note that $\lambda_{\min }$ is usually assumed to be bounded away from zero. In particular, the ERC $M<1$ requires $\lambda_{\text {min }}>0$. The following lemma shows that $\lambda_{\min }$ and $\lambda_{\max }$ can also be bounded in terms of $\mu$. A similar, but slightly weaker result was given in Needell and Tropp (2008).

Lemma 2 Suppose $\mu<\frac{1}{k-1}$, then $1-(k-1) \mu \leq \lambda_{\min } \leq \lambda_{\max } \leq 1+(k-1) \mu$, where $k$ denotes the cardinality of $T$.

It is easy to see that, in order for any variable selection procedure to work properly, both the degree of collinearity among the columns of $X$ and the signal-to-noise ratio need to be properly controlled. Generally speaking, to recover accurately the support of the unknown signal, the degree of linear dependency among the $X_{i}$ 's needs to be small, otherwise the effects of the variables cannot be well separated. On the other hand, the signal-to-noise ratio needs to be sufficiently high in order for the significant variables to be selected. In the case of OMP, the performance of the algorithm depends on the probability of selecting 
a correct variable at each step. This probability is affected by the degree of collinearity among the variables and the noise structure.

We shall begin with the bounded noise cases and then consider the Gaussian case. As mentioned in Section 2, two types of bound noise are considered: $\|\epsilon\|_{2} \leq b_{2}$ and $\left\|X^{\prime} \epsilon\right\|_{\infty} \leq$ $b_{\infty}$. Once the bounded noise cases are understood, the Gaussian case follows easily. In what follows, our analysis of the OMP algorithm will be carried out in terms of the mutual incoherence $\mu$. However, all the main results also hold under the ERC with essentially the same proofs. We shall focus on the MIP in the next section and discuss the results under the ERC $M<1$ in Section 4.

\section{1 $\ell_{2}$ Bounded Noise}

We first consider the case where the error vector $\epsilon$ is bounded in $\ell_{2}$ norm with $\|\epsilon\|_{2} \leq b_{2}$. In this case we set the stopping rule as $\left\|r_{i}\right\|_{2} \leq b_{2}$. It is intuitively easy to see that this rule is reasonable because in the special case of $\beta \equiv 0$ the stopping rule will guarantee that OMP does not select any incorrect variables. We have the following result for OMP with this stopping rule.

Theorem 1 Suppose $\|\epsilon\|_{2} \leq b_{2}$ and $\mu<\frac{1}{2 k-1}$. Then the OMP algorithm with the stopping rule $\left\|r_{i}\right\|_{2} \leq b_{2}$ recovers exactly the true subset of correct variables $X(T)$ if all the nonzero coefficients $\beta_{i}$ satisfy $\left|\beta_{i}\right| \geq \frac{2 b_{2}}{1-(2 k-1) \mu}$.

Theorem 1 and other main results given in this paper can also be stated under the ERC $M<1$. We formally restate Theorem 1 under the ERC below and only make brief remarks for the other results later. See Section 4 for more discussions.

Proposition 1 Suppose $\|\epsilon\|_{2} \leq b_{2}$ and $M<1$. Then the OMP algorithm with the stopping rule $\left\|r_{i}\right\|_{2} \leq b_{2}$ recovers exactly the true subset of correct variables $X(T)$ if all the nonzero coefficients $\beta_{i}$ satisfy $\left|\beta_{i}\right| \geq \frac{2 b_{2}}{(1-M) \lambda_{\min }}$. 
This follows from essentially the same argument as the proof of Theorem 1 given in Section 6 .

It is worth noting that after the OMP algorithm returns the true subset $X(T)$, the signal $\beta$ can be easily estimated, for example, by using the ordinary least squares regression on the subset of variables $X(T)$.

Theorem 1 has two conditions, $\mu<\frac{1}{2 k-1}$ and $\left|\beta_{i}\right| \geq \frac{2 b_{2}}{1-(2 k-1) \mu}$, which together ensure the OMP algorithm to recover exactly the true support of the signal. The condition $\mu<\frac{1}{2 k-1}$ was shown to be sharp in the noisy case in Cai, Wang and Xu (2010a). The other condition $\left|\beta_{i}\right| \geq \frac{2 b_{2}}{1-(2 k-1) \mu}$ for all nonzero coefficient $\beta_{i}$ is to ensure that all significant variables are selected.

In many applications, the focus is often on identifying coordinates of $\beta$ with large magnitude or equivalently variables with significant effects, instead of the often too ambitious goal of recovering the whole support of $\beta$ exactly. So a practically interesting question is: Can OMP identify coordinates with large magnitude when some of the nonzero coordinates $\beta_{i}$ are small? The following result shows that the OMP algorithm with the same stopping rule will still select all the most important variables before it possibly also selects incorrect ones.

Theorem 2 Suppose $\|\epsilon\|_{2} \leq b_{2}$ and $\mu<\frac{1}{2 k-1}$. Let

$$
S=\left\{X_{i}: 1 \leq i \leq p,\left|\beta_{i}\right| \geq \frac{2 \sqrt{k} b_{2}}{1-(2 k-1) \mu}\right\} .
$$

Then the OMP algorithm with the stopping rule $\left\|r_{i}\right\|_{2} \leq b_{2}$ selects a correct variable at each step until all the variables in $S$ are selected.

Remark 1 Similar to Theorem 1, Theorem 2 can also be stated under the ERC with the condition $\mu<\frac{1}{2 k-1}$ replaced by $M<1$, and the condition on the minimum magnitude of $\beta_{i}$ in the set $S$ replaced by $\left|\beta_{i}\right|>\frac{2 \sqrt{k} b_{2}}{(1-M) \lambda_{\min }}$. See Section 4 for more discussions. 
In many applications, it is often desirable to select a subset of the support of the signal $\beta$ without incorrectly selecting any coordinates outside of the support. The OMP algorithm with the stopping rule $\left\|r_{i}\right\|_{2} \leq b_{2}$ does not rule out the possibility of incorrectly selecting a zero coordinate after all the significant ones are selected. The OMP with a modified stopping rule can ensure that no zero coordinates are selected. We have the following result.

Theorem 3 Suppose $\|\epsilon\|_{2} \leq b_{2}$ and $\mu<\frac{1}{2 k-1}$. Let

$$
S=\left\{X_{i}: 1 \leq i \leq p,\left|\beta_{i}\right| \geq\left(\frac{(1+(k-1) \mu) 2 \sqrt{k}}{(1-(k-1) \mu)(1-(2 k-1) \mu)}+\frac{2}{1-(k-1) \mu}\right) b_{2}\right\} .
$$

Then OMP with the stopping rule $\left\|r_{i}\right\|_{2} \leq\left(1+\frac{(1+(k-1) \mu) 2 \sqrt{k}}{1-(2 k-1) \mu}\right) b_{2}$ selects a subset $\hat{T}$ such that $S \subset \hat{T} \subset T$.

Hence, all the significant variables in $S$ are selected by the algorithm and all the selected coordinates are in the support of $\beta$.

\section{$3.2 \ell_{\infty}$ Bounded Noise}

We now turn to the case where the noise $\epsilon$ is assumed to satisfying $\left\|X^{\prime} \epsilon\right\|_{\infty} \leq b_{\infty}$. The stopping rule in this case is $\left\|X^{\prime} r_{i}\right\|_{\infty} \leq b_{\infty}$. Similar to the previous case this is a natural stopping rule which ensures that no incorrect variables are selected in the special case of $\beta \equiv 0$. We have the following result for OMP with this stopping rule.

Theorem 4 Suppose $\left\|X^{\prime} \epsilon\right\|_{\infty} \leq b_{\infty}$ and $\mu<\frac{1}{2 k-1}$. Moreover, assume all the nonzero coefficients $\beta_{i}$ satisfy

$$
\left|\beta_{i}\right| \geq \frac{2 b_{\infty}}{1-(2 k-1) \mu}\left(1+\frac{\sqrt{k}}{\sqrt{1-(k-1) \mu}}\right)
$$

Then OMP with the stopping rule $\left\|X^{\prime} r_{i}\right\|_{\infty} \leq b_{\infty}$ will return the true subset $X(T)$. 
Remark 2 Note that $\mu<\frac{1}{2 k-1}$ implies $(k-1) \mu<\frac{1}{2}$. So a special case of the previous theorem is that when

$$
\left|\beta_{i}\right| \geq \frac{2(1+\sqrt{2 k}) b_{\infty}}{1-(2 k-1) \mu}
$$

the OMP algorithm selects the true subset of significant variables $X(T)$.

As in the $\ell_{2}$ bounded noise case, when some of the nonzero coordinates are small, OMP can also identify all the large components in this case. To be more precise, we have the following result.

Theorem 5 Suppose $\left\|X^{\prime} \epsilon\right\|_{\infty} \leq b_{\infty}$ and $\mu<\frac{1}{2 k-1}$. Let

$$
S=\left\{X_{i}: 1 \leq i \leq p,\left|\beta_{i}\right| \geq \frac{2 \sqrt{k} b_{\infty}}{1-(2 k-1) \mu}\left(1+\frac{\sqrt{k}}{\sqrt{1-(k-1) \mu}}\right)\right\} .
$$

Then the OMP algorithm selects a correct variable at each step until all the variables in $S$ are selected.

In addition, with a modified stopping rule, OMP can also ensure that no incorrect variables are selected in this case.

Theorem 6 Suppose $\left\|X^{\prime} \epsilon\right\|_{\infty} \leq b_{\infty}$ and $\mu<\frac{1}{2 k-1}$. Let

$$
S=\left\{X_{i}: 1 \leq i \leq p,\left|\beta_{i}\right| \geq\left(\frac{6 k}{1-(2 k-1) \mu}+4 \sqrt{k}\right)(1+\sqrt{2 k}) b_{\infty}\right\} .
$$

Then OMP with the stopping rule

$$
\left\|X^{\prime} r_{i}\right\|_{\infty} \leq\left(1+\frac{2 \sqrt{k}(1+(k-1) \mu)}{1-(2 k-1) \mu}\right) C b_{\infty},
$$

selects a subset $\hat{T}$ such that $S \subset \hat{T} \subset T$, where the constant $C$ is given by $C=1+\frac{\sqrt{k}}{\sqrt{1-(k-1) \mu}}$.

Remark 3 It will be shown that in fact a stronger result holds. Theorem 6 is true with the set $S$ enlarged to

$$
S=\left\{X_{i}: 1 \leq i \leq p,\left|\beta_{i}\right| \geq\left(\frac{2 k(1+(k-1) \mu)}{(1-(k-1) \mu)(1-(2 k-1) \mu)}+\frac{2 \sqrt{k}}{1-(k-1) \mu}\right) C b_{\infty}\right\},
$$

where $C=1+\frac{\sqrt{k}}{\sqrt{1-(k-1) \mu}}$. 


\subsection{Gaussian Noise}

The Gaussian noise case is of particular interest in statistics. The results on the bounded noise cases given earlier are directly applicable to the case where noise is Gaussian. This is due to the fact that Gaussian noise is "essentially bounded".

Suppose now the noise vector $\epsilon$ follows Gaussian distribution, $\epsilon \sim N\left(0, \sigma^{2} I_{n}\right)$. Define two bounded sets

$B_{2}=\left\{\epsilon:\|\epsilon\|_{2} \leq \sigma \sqrt{n+2 \sqrt{n \log n}}\right\} \quad$ and $\quad B_{\infty}(\eta)=\left\{\epsilon:\left\|X^{T} \epsilon\right\|_{\infty} \leq \sigma \sqrt{2(1+\eta) \log p}\right\}$

where $\eta \geq 0$. The following result, which follows from standard probability calculations, shows that the Gaussian noise $z$ is essentially bounded. The readers are referred to Cai, $\mathrm{Xu}$ and Zhang (2009) for a proof.

Lemma 3 The Gaussian error $\epsilon \sim N\left(0, \sigma^{2} I_{n}\right)$ satisfies

$$
P\left(\epsilon \in B_{2}\right) \geq 1-\frac{1}{n} \quad \text { and } \quad P\left(\epsilon \in B_{\infty}(\eta)\right) \geq 1-\frac{1}{2 p^{\eta} \sqrt{\pi \log p}}
$$

The following result is a direct consequence of the results for $\ell_{2}$ bounded noise case.

Theorem 7 Suppose $\epsilon \sim N\left(0, \sigma^{2} I_{n}\right), \mu<\frac{1}{2 k-1}$ and all the nonzero coefficients $\beta_{i}$ satisfy

$$
\left|\beta_{i}\right| \geq \frac{2 \sigma \sqrt{n+2 \sqrt{n \log n}}}{1-(2 k-1) \mu}
$$

Then OMP with the stopping rule $\left\|r_{i}\right\|_{2} \leq \sigma \sqrt{n+2 \sqrt{n \log n}}$ selects the true subset $X(T)$ with probability at least $1-1 / n$.

One can also directly apply the results for $\ell_{\infty}$ bounded noise case to the Gaussian case. In fact, a stronger result holds.

Theorem 8 Suppose $\epsilon \sim N\left(0, \sigma^{2} I_{n}\right), \mu<\frac{1}{2 k-1}$ and all the nonzero coefficients $\beta_{i}$ satisfy

$$
\left|\beta_{i}\right| \geq \frac{2 \sigma \sqrt{2(1+\eta) \log p}}{1-(2 k-1) \mu}
$$


for some $\eta \geq 0$. Then OMP with the stopping rule $\left\|X^{\prime} r_{i}\right\|_{\infty} \leq \sigma \sqrt{2(1+\eta) \log p}$ selects exactly the correct subset $X(T)$ with probability at least $1-k / p^{\eta} \sqrt{2 \log p}$.

Remark 4 The conditions in the previous Theorem can also be reformulated in terms of $M$ and $\lambda_{\min }$. Suppose $M<1$ and $\lambda_{\min }>0$, then the OMP algorithm can recovery the true support of $\beta$ with high probability when each nonzero coefficient $\beta_{i}$ satisfies

$$
\left|\beta_{i}\right| \geq \frac{2 \sigma \sqrt{2(1+\eta)) \log p}}{(1-M) \lambda_{\min }}
$$

Remark 5 After the OMP algorithm returns the estimated subset, one can use the ordinary least squares to further estimate the values of the nonzero coordinates of $\beta$. Then with high probability, mean squared error of the resulting estimator will be the same as the case when the true support of $\beta$ were known.

It is interesting to compare the results given above with some of the known results in the literature based on other methods. As mentioned in the introduction, $\ell_{1}$ minimization methods are widely used for reconstructing a sparse signal as well as for support recovery. In particular, Zhao and Yu (2006) considered the model selection consistency of the Lasso and introduced the Irrepresentable Condition. First, it is worth noting that if the Irrepresentable Condition holds for every $k$-sparse signal $\beta \in \mathbb{R}^{p}$, then it is equivalent to the ERC. This can be explained as follows. The Irrepresentable Condition requires $\left\|X(U)^{\prime} X(T)\left(X(T)^{\prime} X(T)\right)^{-1} \operatorname{sign}(\beta(T))\right\|_{\infty}<1$, where $U=\left\{i: \beta_{i}=0\right\}$ and $\beta(T)$ denotes the $k$ dimensional subvector that only keeps the nonzero coordinates of $\beta$. If the Irrepresentable Condition holds for every $\beta(T) \in \mathbb{R}^{k}$, then the sum of absolute value of each column of the matrix $\left(X(T)^{\prime} X(T)\right)^{-1} X(T)^{\prime} X(U)$ must be less than 1 , which is equivalent to the ERC. Also, for the Lasso estimator to be sign consistent, the minimum eigenvalue $\lambda_{\text {min }}$ must be positive as we remarked earlier. In Zhao and Yu (2006), the order of magnitude of all the nonzero coefficients $\beta_{i}$ are required to be at least $n^{(1+c) / 2}$ for some $c>0$. This 
condition is much stronger than Condition (6) that is required in Theorem 8 or Condition (7) under the ERC. It is also stronger than Condition (5) used in Theorem 7.

If not all nonzero coordinates of $\beta$ are large, then the OMP algorithm can still select all the significant coordinates of $\beta$ with high probability. More specifically, we have the following result.

Theorem 9 Suppose $\epsilon \sim N\left(0, \sigma^{2} I_{n}\right), \mu<\frac{1}{2 k-1}$ and let

$$
S=\left\{X_{i}: 1 \leq i \leq p,\left|\beta_{i}\right| \geq \frac{2 \sqrt{k} \sigma \sqrt{2(1+\eta) \log p}}{1-(2 k-1) \mu}\right\} .
$$

Then the OMP algorithm selects a correct variable at each step with probability at least $1-1 / p^{\eta} \sqrt{2 \log p}$ until all the variables in $S$ are selected.

As mentioned earlier, it is sometimes desirable to select a subset of the significant variables without selecting any incorrect variables. By modifying the stopping rule in the Gaussian noise case, it is possible to ensure that with high probability OMP only selects the significant variables and does not select incorrect variables. More specifically, we have the following theorem.

Theorem 10 Suppose $\epsilon \sim N\left(0, \sigma^{2} I_{n}\right), \mu<\frac{1}{2 k-1}$ and let

$$
S=\left\{X_{i}: 1 \leq i \leq p,\left|\beta_{i}\right| \geq\left(\frac{6 k}{1-(2 k-1) \mu}+4 \sqrt{k}\right)(1+\sqrt{2 k}) \sqrt{2(1+\eta) \log p}\right\} .
$$

Then the OMP algorithm returns a subset $\hat{T}$ such that $S \subset \hat{T} \subset T$ with probability at least $1-1 / p^{\eta} \sqrt{2 \log p}$

\section{Discussions}

The analysis of the OMP algorithm given in Section 3 is given under the MIP condition $\mu<\frac{1}{2 k-1}$. As mentioned earlier, the main results can all be reformulated under the ERC 
$M<1$. The reason we use the MIP condition is that the mutual incoherence $\mu$ is a computable quantity, while $M$ is not as it depends on the unknown support $T$. A precise restatement of Theorem 1 under the ERC was given in Proposition 1 and a brief comment was given for Theorem 2. We now discuss other results under the ERC.

Theorem 3 holds under the ERC and the result can be restated as follows. Suppose $\|\epsilon\|_{2} \leq b_{2}$ and $M<1$. Let

$$
S=\left\{X_{i}: 1 \leq i \leq p,\left|\beta_{i}\right| \geq\left(\frac{2 \sqrt{k} \lambda_{\max }}{(1-M) \lambda_{\min }^{2}}+\frac{2}{\lambda_{\min }}\right) b_{2}\right\} .
$$

Then the OMP algorithm with the stopping rule $\left\|r_{i}\right\|_{2} \leq\left(1+\frac{2 \sqrt{k} \lambda_{\max }}{(1-M) \lambda_{\min }}\right) b_{2}$ selects a subset $\hat{T}$ such that $S \subset \hat{T} \subset T$. Similar to Theorem 1, Theorem 4 is also true if the MIP condition $\mu<\frac{1}{2 k-1}$ is replaced by $M<1$ and the lower bound on the magnitude of the nonzero $\beta_{i}$ is changed to $\left|\beta_{i}\right| \geq \frac{2(1+\sqrt{2 k}) b_{\infty}}{(1-M) \lambda_{m i n}}$. Other main results can also be restated in terms of the ERC in a similar way.

It is useful to compare our results with some of the known results in the literature. Donoho, Elad and Temlyakov (2006) considered the OMP algorithm for the noiseless and $\ell_{2}$ bounded noise cases. It was shown that OMP can recover the support of the signal when $\mu \leq \frac{1}{2 k-1}\left(1-\frac{2 b_{2}}{\beta_{\text {min }}}\right)$, where $\beta_{\text {min }}=\min _{i}\left\{\left|\beta_{i}\right|: \beta_{i} \neq 0\right\}$, whereas only $\mu<\frac{1}{2 k-1}$ is required in all of our results. As shown in Cai, Wang and Xu (2010a) the condition $\mu<\frac{1}{2 k-1}$ is sharp in the sense that there exists a design matrix $X$ with the mutual incoherence $\mu=\frac{1}{2 k-1}$ such that certain $k$-sparse signals are not identifiable based on $y$ and $X$ in the noiseless case. Moreover, we also considered the case where no lower bound is assumed on the magnitude of the nonzero coordinates of $\beta$. It is shown in this setting that OMP is still able to identify the significant components before possibly selecting the incorrect ones.

Zhang (2009) considered model selection consistency of the OMP algorithm and showed that under suitable stopping conditions, OMP will return a subset of the true support and the number of unselected nonzero components can be bounded. In the present paper 
we show that under a different stopping rule, OMP not only returns a subset of the true support, but also guarantees that all the significant components are selected. The advantage is that with our stopping rule, the algorithm will not ignore any components with large values. This is an important property for many applications. Moreover, with the same probability of identifying the true support, the lower bound on the magnitude of the nonzero coordinates for our method is smaller than what is required in Zhang (2009). For example, when the probability of identifying the true support is set to be $1-k / p^{\eta} \sqrt{2 \log p}$, then the lower bound of nonzero $\left|\beta_{i}\right|$ is $\frac{2 \sigma \sqrt{2(1+\eta)) \log p}}{(1-M) \lambda_{\min }}$ (see Theorem 8), while the lower bound given in Zhang $(2009)$ is $\frac{3 \sigma \sqrt{2((1+\eta)) \log p+\log (4 \sqrt{2 \log p} / k))}}{(1-M) \lambda_{\min }}$.

Finally, we note that Lounici (2008) considered the properties of the LASSO and Dantzig selector under the MIP conditions. It was showed that when the mutual incoherence is sufficiently small both the LASSO and Dantzig selector have desirable variable selection properties. The MIP condition used in Lounici (2008) is $\mu<\frac{1}{3 k}$ for the Dantzig selector and $\mu<\frac{1}{5 k}$ for the LASSO. In comparison, our condition, $\mu<\frac{1}{2 k-1}$, is clearly weaker than both of them and as we mentioned earlier this condition is sharp. In addition, the analysis given in the present paper on variable selection is much more detailed.

\section{Understanding the OMP Algorithm}

We shall prove all the main results in Section 6. To gain insight on the OMP algorithm and to illustrate the main ideas behind the proofs, it is instructive to provide some technical analysis of the algorithm. The analysis sheds light on how and when the OMP algorithm works properly.

Note that the support $T=\left\{i: \beta_{i} \neq 0\right\}$ and the set of significant or "correct" variables is $X(T)=\left\{X_{i}: i \in T\right\}$. At each step of the OMP algorithm, the residual vector is projected onto the space spanned by the selected variables (columns of $X$ ). Suppose the algorithm 
selects the correct variables at the first $t$ steps and the set of all selected variables at the current step is $X\left(c_{t}\right)$. Then $X\left(c_{t}\right)$ contains $t$ variables and $X\left(c_{t}\right) \subset X(T)$. Recall that $P_{t}=X\left(c_{t}\right)\left(X\left(c_{t}\right)^{\prime} X\left(c_{t}\right)\right)^{-1} X\left(c_{t}\right)^{\prime}$ is the projection operator onto the linear space spanned by the elements of $X\left(c_{t}\right)$. Then the residual after $t$ steps can be written as

$$
r_{t}=\left(I-P_{t}\right) y=\left(I-P_{t}\right) X \beta+\left(I-P_{t}\right) \epsilon \equiv s_{t}+n_{t}
$$

where $s_{t}=\left(I-P_{t}\right) X \beta$ is the signal part of the residual and $n_{t}=\left(I-P_{t}\right) \epsilon$ is the noise part of the residual. Let

$$
M_{t, 1}=\max _{x \in X(T)}\left\{\left|x^{\prime} s_{t}\right|\right\}, \quad M_{t, 2}=\max _{x \in X \backslash X(T)}\left\{\left|x^{\prime} s_{t}\right|\right\}
$$

and

$$
N_{t}=\max _{x \in X}\left\{\left|x^{\prime} n_{t}\right|\right\}
$$

It is clear that in order for OMP to select a correct variable at this step, it is necessary to have $\max _{x \in X(T)}\left\{\left|x^{\prime} r_{t}\right|\right\}>\max _{x \in X \backslash X(T)}\left\{\left|x^{\prime} r_{t}\right|\right\}$. A sufficient condition is $M_{t, 1}-M_{t, 2}>2 N_{t}$. This is because $M_{t, 1}-M_{t, 2}>2 N_{t}$ implies

$$
\max _{x \in X(T)}\left\{\left|x^{\prime} r_{t}\right|\right\} \geq M_{t, 1}-N_{t}>M_{t, 2}+N_{t} \geq \max _{x \in X \backslash X(T)}\left\{\left|x^{\prime} r_{t}\right|\right\}
$$

We first focus on the value of $M_{t, 1}-M_{t, 2}$. The following result is due to Tropp (2004).

Lemma 4 Let $M$ be defined as in (3) and let $M_{t, 1}$ and $M_{t, 2}$ be defined as in (8). Then $M M_{t, 1}>M_{t, 2}$ for all $t$.

Note that $M<1$ is the Exact Recovery Condition. From this lemma, we know that $M_{t, 1}-M_{t, 2}>(1-M) M_{t, 1}$. The previous discussion shows that $M_{t, 1}>\frac{2}{1-M} N_{t}$ is a sufficient condition under which OMP will make a correct decision. Then from Lemma 1 that the condition

$$
M_{t, 1}>2 \frac{1-(k-1) \mu}{1-(2 k-1) \mu} N_{t}
$$


guarantees that the OMP algorithm selects a correct variable at the current step. Let $X\left(u_{t}\right)=X(T) \backslash X\left(c_{t}\right)$ denote the set of significant variables that are yet to be selected and let $\beta\left(u_{t}\right)$ denote the corresponding linear coefficients, then $M_{t, 1}=\left\|X\left(u_{t}\right)^{\prime} s_{t}\right\|_{\infty}$. Note that

$$
M_{t, 1}=\left\|X\left(u_{t}\right)^{\prime} s_{t}\right\|_{\infty}=\left\|X\left(u_{t}\right)^{\prime}\left(I-P_{t}\right) X \beta\right\|_{\infty}=\left\|X\left(u_{t}\right)^{\prime}\left(I-P_{t}\right) X\left(u_{t}\right) \beta\left(u_{t}\right)\right\|_{\infty} .
$$

The following lemma, which is proved in Section 6, can be used to further bound $M_{t, 1}$.

Lemma 5 The minimum eigenvalue of $X(T)^{\prime} X(T)$ is less than or equal to the minimum eigenvalue of $X\left(u_{t}\right)^{\prime}\left(I-P_{t}\right) X\left(u_{t}\right)$. The maximum eigenvalue of $X(T)^{\prime} X(T)$ is greater than or equal to the maximum eigenvalue of $X\left(u_{t}\right)^{\prime}\left(I-P_{t}\right) X\left(u_{t}\right)$.

It then follows immediately that $\left\|X\left(u_{t}\right)^{\prime}\left(I-P_{t}\right) X\left(u_{t}\right) \beta\left(u_{t}\right)\right\|_{2} \geq \lambda_{\text {min }}\left\|\beta\left(u_{t}\right)\right\|_{2}$. Lemma 2 now yields

$M_{t, 1} \geq(k-t)^{-1 / 2}\left\|X\left(u_{t}\right)^{\prime} s_{t}\right\|_{2} \geq(k-t)^{-1 / 2} \lambda_{\min }\left\|\beta\left(u_{t}\right)\right\|_{2} \geq(k-t)^{-1 / 2}(1-(k-1) \mu)\left\|\beta\left(u_{t}\right)\right\|_{2}$.

This and equation (9) show that a sufficient condition for selecting a correct variable at the current step is

$$
\left\|\beta\left(u_{t}\right)\right\|_{2}>\frac{2 \sqrt{k-t} N_{t}}{1-(2 k-1) \mu}
$$

Or mode generally,

$$
\left\|\beta\left(u_{t}\right)\right\|_{2}>\frac{2 \sqrt{k-t} N_{t}}{(1-M) \lambda_{\min } \mu}
$$

This means that if any of remaining coefficients is large enough, then OMP will select a correct variable at this step. For example, if there exists an unselected variable $\beta_{i}$ with $\left|\beta_{i}\right|>\frac{2 \sqrt{k} N_{t}}{1-(2 k-1) \mu}$, then a correct variable would be selected. Also, if all the remaining coefficients are relatively large, i.e. $\left|\beta_{i}\right|>\frac{2 N_{t}}{1-(2 k-1) \mu}$ for all $i \in T$, then (10) is satisfied and OMP will select a correct variable at this step. The value of $N_{t}$ depends on the noise structure and different bounds will be used for different cases in Section 6 . 


\section{Proofs}

In this section we shall prove the main results in the order of Theorems $1,3,4,6$, and 8 . The proofs of the other theorems are similar and are thus omitted. Some of the technical lemmas are proved at the end.

\subsection{Proof of Theorem 1}

It follows from the assumption $\|\epsilon\|_{2} \leq b_{2}$ that

$$
\left\|n_{t}\right\|_{2}=\left\|\left(I-P_{t}\right) \epsilon\right\|_{2} \leq\|\epsilon\|_{2} \leq b_{2}
$$

Let $X_{i}$ be any column of $X$. Then

$$
\left|X_{i}^{\prime} n_{t}\right| \leq\left\|X_{i}\right\|_{2}\left\|n_{t}\right\|_{2} \leq b_{2}
$$

This means $N_{t} \leq b_{2}$. It follows from (10) that for any $t<k,\left\|\beta\left(u_{t}\right)\right\|_{2}>\frac{2 \sqrt{k-t} N_{t}}{1-(2 k-1) \mu}$ implies that a correct variable will be selected at this step. So $\left|\beta_{i}\right| \geq \frac{2 b_{2}}{1-(2 k-1) \mu}$ for all nonzero coefficients $\beta_{i}$ ensures that all the $k$ correct variables will be selected in the first $k$ steps.

Let us now turn to the stopping rule. Let $P_{k}$ denote the projection onto the linear space spanned by $X(T)$. Then $\left\|\left(I-P_{k}\right) \epsilon\right\|_{2} \leq\|\epsilon\|_{2} \leq b_{2}$. So when all the $k$ correct variables are selected, the $\ell_{2}$ norm of the residual will be less than $b_{2}$, and hence the algorithm stops. It remains to be shown that the OMP algorithm does not stop early.

Suppose the algorithm has run $t$ steps for some $t<k$. We will verify that $\left\|r_{t}\right\|_{2}>b_{2}$ and so OMP does not stop at the current step. Again, let $X\left(u_{t}\right)$ denote the set of unselected but correct variable and $\beta\left(u_{t}\right)$ be the corresponding coefficients. Note that

$$
\begin{aligned}
\left\|r_{t}\right\|_{2} & =\left\|\left(I-P_{t}\right) X \beta+\left(I-P_{t}\right) \epsilon\right\|_{2} \\
& \geq\left\|\left(I-P_{t}\right) X \beta\right\|_{2}-\left\|\left(I-P_{t}\right) \epsilon\right\|_{2} \geq\left\|\left(I-P_{t}\right) X\left(u_{t}\right) \beta\left(u_{t}\right)\right\|_{2}-b_{2}
\end{aligned}
$$


It follows from Lemma 5 that

$$
\left\|\left(I-P_{t}\right) X\left(u_{t}\right) \beta\left(u_{t}\right)\right\|_{2} \geq \lambda_{\min }\left\|\beta\left(u_{t}\right)\right\|_{2} \geq(1-(k-1) \mu) \frac{2 b_{2}}{1-(2 k-1) \mu}>2 b_{2} .
$$

So

$$
\left\|r_{t}\right\|_{2} \geq\left\|\left(I-P_{t}\right) X\left(u_{t}\right) \beta\left(u_{t}\right)\right\|_{2}-b_{2}>b_{2}
$$

and the theorem is proved.

\subsection{Proof of Theorem 3}

From the proof of Theorem 1, we know that

$$
\left\|r_{t}\right\|_{2} \geq \lambda_{\min }\left\|\beta\left(u_{t}\right)\right\|_{2}-b_{2} \geq(1-(k-1) \mu)\left\|\beta\left(u_{t}\right)\right\|_{2}-b_{2} .
$$

On the other hand,

$$
\begin{aligned}
\left\|r_{t}\right\|_{2} & =\left\|\left(I-P_{t}\right) X \beta+\left(I-P_{t}\right) \epsilon\right\|_{2} \leq\left\|\left(I-P_{t}\right) X\left(u_{t}\right) \beta\left(u_{t}\right)\right\|_{2}+b_{2} \\
& \leq \lambda_{\max }\left\|\beta\left(u_{t}\right)\right\|_{2}+b_{2} \leq(1+(k-1) \mu)\left\|\beta\left(u_{t}\right)\right\|_{2}+b_{2} .
\end{aligned}
$$

So

$$
\frac{\left\|r_{t}\right\|_{2}-b_{2}}{1+(k-1) \mu} \leq\left\|\beta\left(u_{t}\right)\right\|_{2} \leq \frac{\left\|r_{t}\right\|_{2}+b_{2}}{1-(k-1) \mu}
$$

Since the stopping rule is to check whether $\left\|r_{i}\right\|_{2} \leq\left(1+\frac{(1+(k-1) \mu) 2 \sqrt{k}}{1-(2 k-1) \mu}\right) b_{2}$, we know that when the stopping rule is not satisfied

$$
\left\|\beta\left(u_{t}\right)\right\|_{2} \geq \frac{2 \sqrt{k} b_{2}}{1-(2 k-1) \mu}
$$

From the previous discussion, this means OMP will select a correct variable at this step. When the stopping rule is satisfied,

$$
\left\|\beta\left(u_{t}\right)\right\|_{2} \leq\left(\frac{(1+(k-1) \mu) 2 \sqrt{k}}{(1-(k-1) \mu)(1-(2 k-1) \mu)}+\frac{2}{1-(k-1) \mu}\right) b_{2},
$$

and so all the variables in the set $S=\left\{X_{i}: 1 \leq i \leq p,\left|\beta_{i}\right| \geq \frac{(1+(k-1) \mu) 2 \sqrt{k}}{(1-(k-1) \mu)(1-(2 k-1) \mu)}+\right.$ $\left.\left.\frac{2}{1-(k-1) \mu}\right) b_{2}\right\}$ have been selected. 


\subsection{Proof of Theorem 4}

Since $\left\|X^{\prime} \epsilon\right\|_{\infty} \leq b_{\infty}$ and $\lambda_{\min } \geq 1-(k-1) \mu$, for any $t<k$

$$
\left\|P_{t} \epsilon\right\|_{2}^{2}=\epsilon^{\prime} X\left(c_{t}\right)\left(X\left(c_{t}\right)^{\prime} X\left(c_{t}\right)\right)^{-1} X\left(c_{t}\right)^{\prime} \epsilon \leq \frac{1}{\lambda_{\min }}\left\|X\left(c_{t}\right)^{\prime} \epsilon\right\|_{2}^{2} \leq \frac{t b_{\infty}^{2}}{1-(k-1) \mu}
$$

Let $X_{i}$ be any column of $X$. Then

$$
\left|X_{i}^{\prime} n_{t}\right|=\left|X_{i}^{\prime}\left(I-P_{t}\right) \epsilon\right| \leq\left|X_{i}^{\prime} \epsilon\right|+\left|X_{i}^{\prime} P_{t} \epsilon\right| \leq b_{\infty}+\frac{\sqrt{t} b_{\infty}}{\sqrt{1-(k-1) \mu}},
$$

which implies

$$
N_{t} \leq\left(1+\frac{\sqrt{t}}{\sqrt{1-(k-1) \mu}}\right) b_{\infty}
$$

Now since

$$
\left|\beta_{i}\right| \geq \frac{2 b_{\infty}}{1-(2 k-1) \mu}\left(1+\frac{\sqrt{k}}{\sqrt{1-(k-1) \mu}}\right)
$$

we have $\left\|\beta\left(u_{t}\right)\right\|_{2}>\frac{2 \sqrt{k-t} N_{t}}{1-(2 k-1) \mu}$, which ensures that OMP selects a correct variable at this step.

We now turn to the stopping rule. It suffices to prove that for any $t<k,\left\|X^{\prime} r_{t}\right\|_{\infty}>b_{\infty}$ and so the algorithm does not stop early. It can be seen that

$$
\begin{aligned}
\left\|X^{\prime} r_{t}\right\|_{\infty} & =\left\|X^{\prime}\left(I-P_{t}\right) X \beta+X^{\prime}\left(I-P_{t}\right) \epsilon\right\|_{\infty} \\
& \geq\left\|X\left(u_{t}\right)^{\prime}\left(I-P_{t}\right) X\left(u_{t}\right) \beta\left(u_{t}\right)\right\|_{\infty}-\left\|X\left(u_{t}\right)^{\prime}\left(I-P_{t}\right) \epsilon\right\|_{\infty} \\
& \geq \frac{1}{\sqrt{k-t}}\left\|X\left(u_{t}\right)^{\prime}\left(I-P_{t}\right) X\left(u_{t}\right) \beta\left(u_{t}\right)\right\|_{2}-\left(1+\frac{\sqrt{t}}{\sqrt{1-(k-1) \mu}}\right) b_{\infty} \\
& \geq \frac{1}{\sqrt{k-t}} \lambda_{\min }\left\|\beta\left(u_{t}\right)\right\|_{2}-\left(1+\frac{\sqrt{t}}{\sqrt{1-(k-1) \mu}}\right) b_{\infty} \\
& \geq\left(1+\frac{\sqrt{t}}{\sqrt{1-(k-1) \mu}}\right) b_{\infty}>b_{\infty}
\end{aligned}
$$

and the theorem then follows. 


\subsection{Proof of Theorem 6}

The proof of this theorem is similar to that of Theorem 3. Note that

$$
\frac{\left\|X^{\prime} r_{t}\right\|_{\infty}-C b_{\infty}}{1+(k-1) \mu} \leq\left\|\beta\left(u_{t}\right)\right\|_{2} \leq \frac{\left\|X^{\prime} r_{t}\right\|_{\infty}+C b_{\infty}}{1-(k-1) \mu} \sqrt{k}
$$

where $C=1+\frac{\sqrt{k}}{\sqrt{1-(k-1) \mu}}$. This ensures that if the stopping rule is not satisfied, i.e.

$$
\left\|X^{\prime} r_{i}\right\|_{\infty}>\left(1+\frac{2 \sqrt{k}(1+(k-1) \mu)}{1-(2 k-1) \mu}\right) C b_{\infty}
$$

then $\left\|\beta\left(u_{t}\right)\right\|_{2}>\frac{2 \sqrt{k} C}{1-(2 k-1) \mu} b_{\infty}$ and so OMP will select a correct variable at this step. On the other hand, when the stopping rule is satisfied, all the variables in

$$
S=\left\{X_{i}: 1 \leq i \leq p,\left|\beta_{i}\right| \geq\left(\frac{2 k(1+(k-1) \mu)}{(1-(k-1) \mu)(1-(2 k-1) \mu)}+\frac{2 \sqrt{k}}{1-(k-1) \mu}\right) C b_{\infty}\right\}
$$

are selected.

\subsection{Proof of Theorem 8}

Firstly, we will prove that with high probability $(1-M) M_{t 1}>2 N_{t}$ at any step $t<k$. It can be seen that

$$
M_{t 1} \geq(k-t)^{-1 / 2}\left\|X\left(u_{t}\right)^{\prime} s_{t}\right\|_{2} \geq(k-t)^{-1 / 2} \lambda_{\min }\left\|\beta\left(u_{t}\right)\right\|_{2}
$$

Since for any $t<k, N_{t}=X^{\prime}\left(I-P_{t}\right) \epsilon$, it follows that

$$
P\left(N_{t} \leq \sigma \sqrt{2(1+\eta) \log p}\right) \geq 1-\frac{1}{p^{\eta} \sqrt{2 \log p}}
$$

This means if

$$
\left\|\beta\left(u_{t}\right)\right\|_{2} \geq \frac{2 \sqrt{k-t} \sigma \sqrt{2(1+\eta) \log p}}{(1-M) \lambda_{\min }}
$$

with probability at least $1-\frac{1}{p^{\eta} \sqrt{2 \log p}},(1-M) M_{t 1}>2 N_{t}$ and hence a correct variable is selected at the current step. This is true when

$$
\left|\beta_{i}\right| \geq \frac{2 \sigma \sqrt{2(1+\eta) \log p}}{(1-M) \lambda_{\min }}
$$


for some $i \in T$. Therefore under the conditions of the theorem, we can select all the $k$ correct variables at the first $k$ steps with probability at least $1-\frac{k}{p^{\eta} \sqrt{2 \log p}}$.

We now consider the stopping rule. Suppose $N_{t} \leq \sigma \sqrt{2(1+\eta) \log p}$ for $t=1,2, \cdots, k$, which means the algorithm makes correct decisions at the first $k$ steps. Now using the same argument as in the proof of Theorem 4, it can be shown that for any $t<k$, when $N_{t} \leq \sigma \sqrt{2(1+\eta) \log p}$, the algorithm will not stop early. And when $t=k$ since $N_{k} \leq \sigma \sqrt{2(1+\eta) \log p}$ and all the correct variables have been selected, the stopping rule is satisfied and hence the algorithm stops. So the probability of selecting exactly the correct subset is at least

$$
\begin{aligned}
& P\left(N_{t} \leq \sigma \sqrt{2(1+\eta) \log p} \text { for } t=1,2, \cdots, k\right) \\
\geq & 1-\sum_{t=1}^{k} P\left(N_{t}>\sigma \sqrt{2(1+\eta) \log p}\right) \geq 1-\frac{k}{p^{\eta} \sqrt{2 \log p}}
\end{aligned}
$$

\subsection{Proofs of the Technical Lemmas}

Proof of Lemma 2: To prove the lower bound on $\lambda_{\text {min }}$, it suffices to show that when $\mu<1 /(k-1)$, the matrix $X(T)^{\prime} X(T)-\lambda I$ is nonsingular for any $\lambda<1-(k-1) \mu$. This is equivalent to show that for any nonzero vector $c=\left(c_{1}, c_{2}, \cdots, c_{k}\right)^{\prime} \in R^{k},\left(X(T)^{\prime} X(T)-\right.$ $\lambda I) c \neq 0$. Without loss of generality, suppose $\left|c_{1}\right| \geq\left|c_{2}\right| \geq \cdots \geq\left|c_{k}\right|$ and $X(T)=$ $\left(X_{T 1}, X_{T 2}, \cdots, X_{T k}\right)$. Then the first coordinate of the vector $\left(X(T)^{\prime} X(T)-\lambda I\right) c$ satisfies

$$
\begin{aligned}
\left|\left\{\left(X(T)^{\prime} X(T)-\lambda I\right) c\right\}_{1}\right| & =\left|(1-\lambda) c_{1}+X_{T 1}^{\prime} X_{T 2} c_{2}+\cdots+X_{T 1}^{\prime} X_{T k} c_{k}\right| \\
& \geq(1-\lambda)\left|c_{1}\right|-\mu\left(\left|c_{2}\right|+\cdots+\left|c_{k}\right|\right) \\
& >(k-1) \mu\left|c_{1}\right|-\mu\left(\left|c_{2}\right|+\cdots+\left|c_{k}\right|\right) \geq 0 .
\end{aligned}
$$

This means $\left(X(T)^{\prime} X(T)-\lambda I\right) c \neq 0$, and hence $\lambda_{\min } \geq 1-(k-1) \mu$ is proved. By the same argument, it can be shown that $\lambda_{\max } \leq 1+(k-1) \mu$. 
Proof of Lemma 5: From the standard result on the inverse of a block matrix, we know that $\left(X\left(u_{t}\right)^{\prime}\left(I-P_{t}\right) X\left(u_{t}\right)\right)^{-1}$ is a main submatrix of $\left(X(T)^{\prime} X(T)\right)^{-1}$. Therefore the maximum eigenvalue of $\left(X\left(u_{t}\right)^{\prime}\left(I-P_{t}\right) X\left(u_{t}\right)\right)^{-1}$ is less than or equal to the maximum eigenvalue of $\left(X(T)^{\prime} X(T)\right)^{-1}$. Also the minimum eigenvalue of $\left(X\left(u_{t}\right)^{\prime}\left(I-P_{t}\right) X\left(u_{t}\right)\right)^{-1}$ is greater than or equal to the minimum eigenvalue of $\left(X(T)^{\prime} X(T)\right)^{-1}$. The lemma then follows.

\section{References}

[1] Bickel, P., Ritov, Y., and Tsybakov, A. (2009). Simultaneous analysis of Lasso and Dantzig selector. Ann. Statist. 37, 1705-1732.

[2] Barron, A., Cohen, A., Dahmen, W. and DeVore, R. (2008). Approximation and learning by greedy algorithms. Ann. Statist. 36, 64-94.

[3] Cai, T., Wang, L., and Xu, G. (2010a). Stable recovery of sparse signals and an oracle inequality. IEEE Trans. Inf. Theory 56, 3516-3522.

[4] Cai, T., Wang, L., and Xu, G.(2010b). New bounds for restricted isometry constants, IEEE Trans. Inf. Theory 56, 4388-4394.

[5] Cai, T., Xu, G., and Zhang, J. (2009). On recovery of sparse signals via $l_{1}$ minimization. IEEE Trans. Inf. Theory. bf 55, 3388-3397.

[6] Candes, E. and Tao, T. (2005). Decoding by linear programming. IEEE Trans. Inf. Theory 51, 4203-4215.

[7] Candes, E. and Tao, T. (2007). The Dantzig selector: statistical estimation when $p$ is much larger than $n$. Ann. Statist. 35, 2313-2351. 
[8] Chen, S. S., Donoho, D. L. and Saunders, M. A. (1998). Atomic decomposition by basis pursuit. SIAM J. Sci. Comput. 20, 33-61.

[9] Davis, G., Mallat, S. and Avellaneda, M. (1997). Greedy adaptive approximation. J. Constr. Approx. 13, 57-98.

[10] Donoho, D.L., Elad, M. and Temlyakov, V.N. (2006). Stable recovery of sparse overcomplete representations in the presence of noise. IEEE Trans. Inf. Theory. 52, 6-18.

[11] Donoho, D. L., and Huo, X. (2001). Uncertainty principles and ideal atomic decomposition, IEEE Trans. Inf. Theory. 47, 2845-2862.

[12] Efron, B., Hastie, T., Johnstone, I. and Tibshirani, R. (2004). Least angle regression (with discussion), Ann. Statist. 32, 407C451.

[13] Lounici, K. (2008). Sup-norm convergence rate and sign concentration property of Lasso and Dantzig estimators. Electronic Journal of Statistics. 2, 90-102.

[14] Needell, D. and Tropp, J.A. (2008). CoSaMP: Iterative signal recovery from incomplete and inaccurate samples. Appl. Comp. Harmonic Anal. 26. 301-321.

[15] Tibshirani, R. (1996). Regression shrinkage and selection via the lasso, J. Roy. Statist. Soc. Ser. B, 58, 267C288.

[16] Tropp, J. A. (2004). Greed is good: Algorithmic results for sparse approximation. IEEE Trans. Inf. Theory 50, 2231-2242.

[17] Tropp, J. A. (2006). Just relax: Convex programming methods for identifying sparse signals. IEEE Trans. Inf. Theory 51, 1030-1051.

[18] Zhang, T. (2009). On the consistency of feature selection using greedy least squares regression. J. Machine Learning Research 10, 555-568. 
[19] Zhao, P. and Yu, B. (2006). On Model Selection Consistency of Lasso. J. Machine Learning Research 7, 2541-2567. 